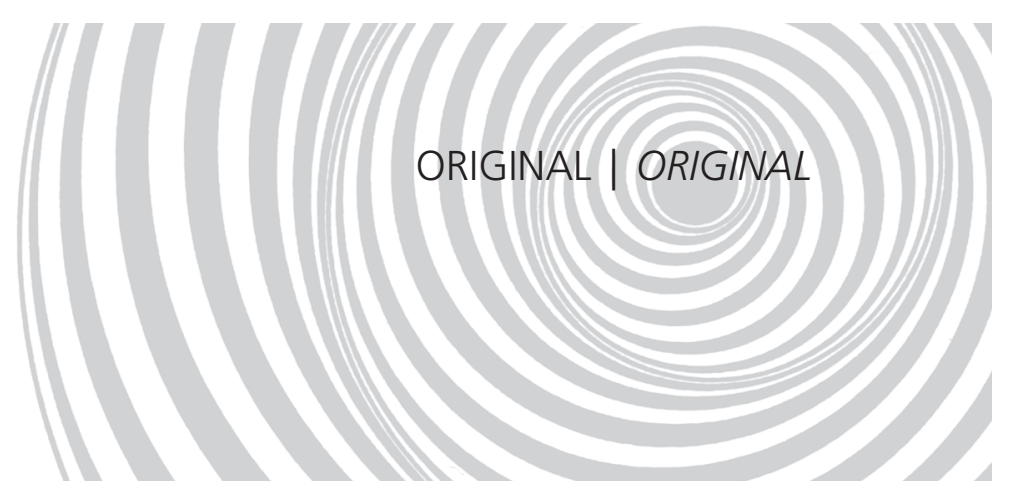

\title{
Fatores associados à duração mediana do aleitamento materno em lactentes nascidos em município do estado de São Paulo'
}

\author{
Factors associated with the median breastfeeding \\ duration of infants born in a city \\ of São Paulo State
}

Julia Laura Delbue BERNARDI $1^{2,3}$

Regina Esteves JORDÃO3

Antônio de Azevedo BARROS FILHO ${ }^{3}$

RES U M O

\section{Objetivo}

Verificar a prevalência do aleitamento materno entre lactentes, correlacionada com variáveis sócio-demográficas, em Campinas (SP).

\section{Métodos}

Entre os anos de 2004 e 2005, entrevistadores visitaram 2857 domicílios de crianças menores de dois anos e aplicaram às mães um questionário estruturado, composto por perguntas sobre nível socioeconômico, amamentação e alimentação complementar. O estudo foi transversal, de base populacional, randomizado, realizado com base nos dados da Declaração de Nascidos Vivos e da prevalência do aleitamento. A duração mediana do aleitamento foi determinada pela análise de sobrevida de Kaplan Méier, e o ajuste do tempo, pela análise multivariada de Cox. A significância estatística adotada foi de $5 \%$.

\section{Resultados}

A mediana do aleitamento materno exclusivo foi de 90 dias (Intervalo de Confiança - IC95\%: 87,6-92,4) e a do aleitamento materno geral foi de 120 dias (IC95\%: 117,7-122,3). Com um mês de vida, 66,2\% das crianças estavam sendo amamentadas exclusivamente com leite do peito, índice que diminuiu para 2,3\% aos seis meses. A introdução mediana para chá (IC95\%: 113,2-126,8) e para leite em pó (IC95\%: 112,7-127,3) foi de 120 dias. A cor da pele da criança, a escolaridade, a profissão e o estado civil maternos foram fatores associados à duração e ao tipo de amamentação.

1 Artigo elaborado a partir da tese de J.L.D. BERNARDI, intitulada "Prevalência do aleitamento materno, introdução complementar de alimentos e crescimento de menores de dois anos em Campinas, São Paulo". Universidade Estadual de Campinas; 2007.

2 Pontifícia Universidade Católica de Campinas, Centro de Ciências da Vida, Faculdade de Nutrição. Av. John Boyd Dunlop, s/n., Prédio Administrativo, Jd. Ipaussurama, 13060-904, Campinas, SP, Brasil. Correspondência para/Correspondence to: J.L.D. BERNARDI. E-mail: <julialdb@fcm.unicamp.br>.

3 Universidade Estadual de Campinas, Faculdade de Ciências Médicas, Departamento de Pediatria. Campinas, SP, Brasil. 
868 | J.L.D. BERNARDI et al.

\section{Conclusão}

Fatores sócio-demográficos podem interferir na duração mediana do aleitamento materno que, embora esteja melhor do que em outros locais do Brasil, está abaixo do padrão recomendado internacionalmente, sugerindo a necessidade de maiores investimentos em relação a este fato, no município.

Termos de indexação: Aleitamento materno. Desmame. Estudos transversais.

\section{A B S T R A C T}

\section{Objective}

The aim of this study was to verify the proportion of breastfed infants and correlate it with sociodemographic data in Campinas (SP), Brazil.

\section{Methods}

Between 2004 and 2005, interviewers visited 2,857 homes of children under two years of age and interviewed their mothers with a structured questionnaire containing questions regarding their socioeconomic level, breastfeeding practices and complementary foods. This randomized, cross-sectional, population-based study was based on data of "Children Born Alive" and on the prevalence of breastfeeding. The median breastfeeding duration was determined by Kaplan Meier's survival analysis and the time was adjusted by Cox's multivariate analysis. The significance level was set at 5\%.

\section{Results}

Median exclusive breastfeeding was 90 days (CI 95\%: 87.6 - 92.4); and median general breastfeeding was 120 days (Cl 95\%: 117.7 - 122.3). At 1 month of age, 66.2\% of the children were exclusively breastfed; this percentage decreased to $2.3 \%$ at 6 months of age. Tea and powdered milk were introduced at a median age of 120 days (Cl 95\%: 113.2 - 126.8 and Cl 95\%: 112.7 - 127.3 respectively). The child's skin color and the mother's education level, profession and marital status were associated with breastfeeding duration and practices.

\section{Conclusion}

Sociodemographic factors can interfere in median breastfeeding duration. Although the median breastfeeding duration in Campinas is better than elsewhere in Brazil, it is below the internationally recommended median, the city needs to invest more in this area.

Indexing terms: Breastfeeding. Weaning. Cross sectional studies.

\section{N T R O D U ÇÃ O}

O Aleitamento Materno (AM) é uma prática biologicamente determinada, mas que sofre influência cultural e alguns fatores como a assistência pré-natal, a entrada da mulher no mercado de trabalho e a industrialização dos leites, podem interferir no seu sucesso ${ }^{1,2}$. Nas últimas décadas, principalmente nos países em desenvolvimento, a valorização desta prática foi retomada propiciando a diminuição da desnutrição, dos processos alérgicos, da diarréia e da anemia ${ }^{3-6}$.

No início dos anos 1980, a Organização Mundial da Saúde (OMS) e o Fundo das Nações Unidas para a Infância (UNICEF) ${ }^{7}$ deram início a uma mobilização mundial para a Promoção e Proteção ao aleitamento materno, por meio de iniciativas como a instituição do Código Inter- nacional de Substitutos do Aleitamento Materno. No Brasil ${ }^{6}$ houve a criação do Instituto Nacional de Alimentação e Nutrição (INAN) e do Programa Nacional de Incentivo ao Aleitamento Materno (PINAM). Nesta época, algumas maternidades iniciaram o processo de Alojamento Conjunto entre mães e recém-nascidos, a implantação do Banco de Leite Humano $(B L H)^{8}$ e dos Grupos de Apoio à Amamentação; além disso, o governo estipulou a licença maternidade para mães trabalhadoras. Na década de 1990 foi criada a Iniciativa do Hospital Amigo da Criança (IHAC) ${ }^{8}$. Neste período, foram instituídos os "Dez passos para o aleitamento materno" e, em 1997, a definição dos novos termos sobre os tipos de aleitamento materno pela OMS 1,9,10: aleitamento materno exclusivo, predo-minante e geral. 
A pesquisa realizada pela Organização Mundial da Saúde", sobre as "Tendências dos Índices de Amamentação 1990 - 2000" mostrou que o percentual de mudança para o Aleitamento Materno Exclusivo (AME), nos primeiros quatro meses de vida das crianças, foi 8,0\% positivo entre os 37 países participantes. Segundo o Ministério da Saúde ${ }^{11}$ e a Fundação SEADE ${ }^{12}$, no ano de 2000 a prevalência do AME no Brasil era de 53,1\% para um mês, $21,6 \%$ entre três e quatro meses e $9,7 \%$ entre cinco e seis meses, enquanto que na cidade de São Paulo estes índices eram de 36,2\%, $15,1 \%$ e $7,6 \%$, respectivamente. Os fatores de risco para a ocorrência do desmame foram a baixa escolaridade materna, a primiparidade, maternidade precoce e o desconhecimento da prática da amamentação. No ano de 2006, a pesquisa sobre Política Nacional de Demografia e Saúde (PNDS) ${ }^{13}$ revelou que 3,6\% das crianças menores de cinco anos que vivem no Brasil, nunca tinham sido amamentadas.

Campinas fica a, aproximadamente, $100 \mathrm{~km}$ da cidade de São Paulo sendo um dos principais pólos tecnológicos, universitários e financeiros do Brasil. Ultrapassou a marca de um milhão de habitantes e possui uma das maiores rendas per capita do País. A cidade conta com adequada estrutura de hospitais e maternidades, que atendem satisfatoriamente às necessidades da rede pública de saúde. Possui taxas reduzidas de mortalidade infantil e programas de incentivo ao aleitamento materno, com duas universidades com maternidades que integram a Iniciativa do Hospital Amigo da Criança.

Apesar destas características, os estudos sobre amamentação no município são escassos e compostos por amostras regionais. Motivado por este aspecto, o objetivo do estudo foi verificar a prevalência do aleitamento materno e os fatores associados à época mediana do desmame, de menores de dois anos moradoras da cidade, com o intuito de obter uma visão panorâmica da situação do aleitamento materno na cidade, contribuindo para o melhor direcionamento dos programas de atenção à saúde das crianças.

\section{MÉ T O D O S}

O município de Campinas trabalha com a Declaração de Nascidos Vivos (SINASC), que é um registro de banco de dados de nascimentos ocorridos mensalmente, sob coordenação da Secretaria de Estado da Saúde. Em uma planilha são fornecidos o número de consultas de pré-natal, a idade gestacional, o sexo, a etnia (cor da pele da criança informada pela mãe), o peso, o comprimento, o escore Apgar da criança, a idade materna por ocasião do parto, o tipo de parto e o endereço do responsável. As maternidades enviam informações para o SINASC, que disponibiliza seus dados mediante solicitação, sendo recomendado e validado para pesquisas de campo ${ }^{14}$.

O estudo foi do tipo transversal, de base populacional, randomizado, feito por sorteio no qual os lactentes foram selecionados com base na declaração de nascidos vivos de Campinas (SINASC). Utilizou-se o cálculo da amostra para proporção de população finita tendo, como fontes de informação, os dados de nascimento e a prevalência do aleitamento materno no município, descritos em anos anteriores. De acordo com o mês de nascimento, realizou-se um sorteio e, optando-se pelo erro amostral de 0,065\%, foi fixado o tamanho da população em 2747 lactentes sendo sorteados 3 mil e 2857 participaram, contemplando o número calculado. Primeiramente, foram avaliadas as crianças com idade entre 24 e 13 meses e, posteriormente, aquelas com idade entre 12 e 1 mês (Figura 1).

Entre os meses de maio de 2004 e maio de 2005, de forma ininterrupta, foram visitados 2857 domicílios de crianças menores de dois anos, e as mães foram entrevistadas em suas casas por pessoas devidamente treinadas, respeitando-se o bem estar físico, moral e social dos participantes. As informações foram obtidas prioritariamente com a mãe da criança e, quando esta não era localizada, novas tentativas eram feitas. Após três visitas, o questionário era respondido por outro responsável, ou descartado e substituído por um novo sorteio. Os profissionais (nove nutricionistas 


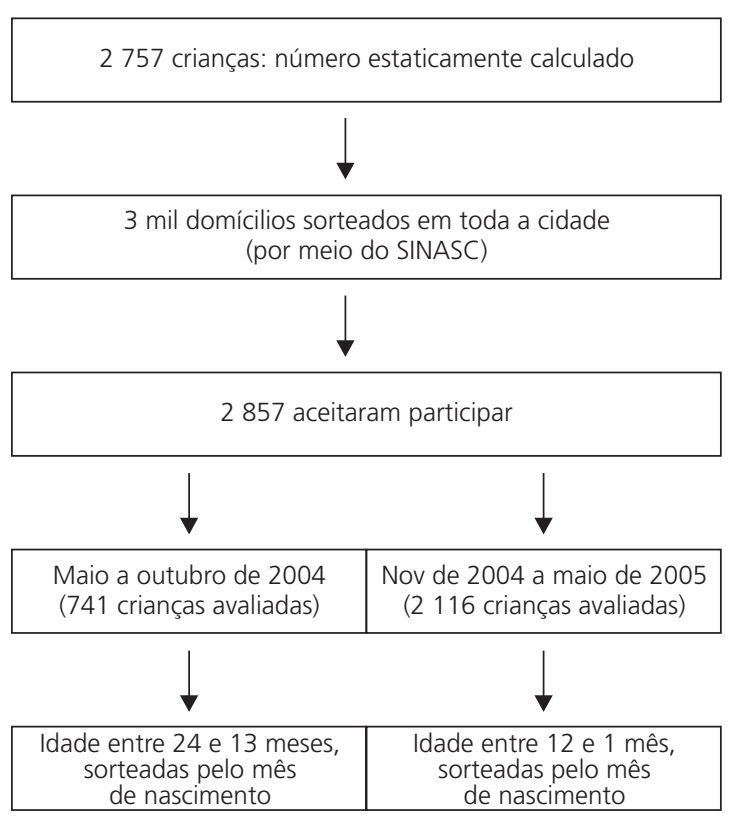

Figura 1. Distribuição das crianças por faixa etária e mês de avaliação pelos entrevistadores domiciliares.

e um técnico de enfermagem) receberam treinamento quanto à utilização dos instrumentos a serem aplicados na pesquisa, nos três meses anteriores, testando, na prática, a técnica da entrevista e o manejo do questionário. Todo o processo foi acompanhado pelos pesquisadores e por um supervisor de campo, que gerenciou todas as etapas.

O questionário estruturado era composto por perguntas fechadas contendo informações sócio-econômico-demográficas sobre as condições de moradia, a profissão materna (do lar, nível superior, superior da área da saúde - médica, psicóloga, nutricionista, fonoaudióloga, fisioterapeuta, terapeuta ocupacional, enfermeira, cirurgiã dentista, farmacêutica -, nível técnico ou de baixa escolaridade e desempregada no momento da entrevista), o nível de escolaridade (categorizado pelos anos de estudo), a distribuição da renda (em salários-mínimos), o número de itens domésticos (de zero a quatro bens: geladeira, freezer, fogão, TV a cores e preto e branco, rádio, telefone fixo e celular, computador e acesso a internet). Estes dados foram usados como um marco sobre o nível sócioeconômico e de condições de vida da criança e de sua família"1.
Em relação à amamentação, foram realizadas perguntas sobre a primeira mamada, tipo de aleitamento oferecido no momento da entrevista, dificuldades maternas relacionadas à amamentação, época do desmame e tempo de aleitamento, tipo de leite oferecido, introdução de água, leites comerciais e chá. Para a avaliação do tempo do aleitamento estipularam-se as categorias do aleitamento materno estabelecidas pela Organização Mundial da Saúde 1,9,10: Exclusivo (AME), Predominante (AMP) e Geral (AM). Define-se como Aleitamento Exclusivo, quando a criança recebe somente leite de peito diretamente da mama ou extraído, sem adição de nenhum outro líquido ou sólido, com exceção de gotas ou xaropes de vitaminas, minerais e medicamentos; Predominante, quando há, além do leite da mãe, inclusão de líquidos como água, chá e sucos de fruta; por fim, no Aleitamento Materno Geral, a criança recebe leite materno associado a alimentos sólidos e semi-sólidos, incluindo o leite não-humano.

Foram excluídas do estudo as crianças prematuras, as nascidas de baixo peso, as maiores de dois anos, as portadoras de doenças graves que pudessem interferir no processo de crescimento, tais como síndromes neurológicas e doença pulmonar na forma grave, as adotadas e as não moradoras do endereço.

Para descrever o perfil da amostra foram feitas as tabelas de freqüência das variáveis categóricas com os valores de freqüência absoluta (n) e percentual (\%) e estatísticas descritivas das variáveis contínuas com valores de mediana. Para analisar o tempo de desmame foi utilizada a análise de sobrevida pelo método de estimativa de Kaplan Méier. O modelo de regressão de Cox (critério Stepwise) foi utilizado para obter o ajuste relacionado ao tempo do aleitamento. O nível de significância estatística adotado para todos os testes foi de $5 \%(p<0,05)$. Os dados foram analisados em microcomputador, utilizando-se os programas Epi info ${ }^{15}$ versão 6.04b e o SPSS ${ }^{16}$. 
A participação no estudo foi voluntária e possível somente após o preenchimento do Termo de Consentimento Livre e Esclarecido pelos responsáveis. O trabalho foi aprovado pelo Comitê de Ética em Pesquisa da Universidade Estadual de Campinas (CEP 533/2002).

Tabela 1. Distribuição das variáveis sócio-demográficas da amostra de 2857 crianças estudadas em 2004-2005. Campinas (SP), 2004-2005.

\begin{tabular}{|c|c|c|}
\hline \multirow{2}{*}{ Variáveis } & \multicolumn{2}{|c|}{ Amostra } \\
\hline & $n$ & $\%$ \\
\hline \multicolumn{3}{|l|}{ Cor da pele da criança } \\
\hline Branca & 2117 & 74,0 \\
\hline Parda & 610 & 21,4 \\
\hline Negra & 105 & 3,7 \\
\hline Amarela & 25 & 0,9 \\
\hline \multicolumn{3}{|l|}{ Idade materna } \\
\hline Menos de 20 anos & 387 & 13,5 \\
\hline De 20 a 34 anos & 2110 & 73,9 \\
\hline Mais de 35 anos & 360 & 12,6 \\
\hline \multicolumn{3}{|l|}{ Escolaridade materna } \\
\hline 0 a 4 anos & 222 & 7,8 \\
\hline 5 a 8 anos & 814 & 28,5 \\
\hline 9 a 11 anos & 1278 & 44,7 \\
\hline Mais de 12 anos & 543 & 19,0 \\
\hline \multicolumn{3}{|l|}{ Profissão materna } \\
\hline Do lar & 1155 & 40,4 \\
\hline Nível superior & 114 & 4,0 \\
\hline Superior da área da saúde* & 77 & 2,7 \\
\hline Nível técnico & 1310 & 45,9 \\
\hline Desempregada no momento & 201 & 7,0 \\
\hline \multicolumn{3}{|l|}{ Renda familiar (salários-mínimos) } \\
\hline Menos de 2 & 639 & 22,4 \\
\hline De 2 a 2,9 & 764 & 26,7 \\
\hline De 3 a 5,9 & 729 & 25,5 \\
\hline De 6 a 9,9 & 277 & 9,7 \\
\hline$\geq 10$ & 448 & 15,7 \\
\hline \multicolumn{3}{|c|}{ Número total de bens domésticos** } \\
\hline 0 a 4 & 560 & 19,6 \\
\hline 5 a 9 & 1660 & 58,1 \\
\hline 10 a 14 & 525 & 18,4 \\
\hline 15 a 19 & 103 & 3,6 \\
\hline$\geq 20$ & 9 & 0,3 \\
\hline
\end{tabular}

* Profissão materna (superior da área da saúde: médica, psicóloga, nutricionista, fonoaudióloga, fisioterapeuta, terapeuta ocupacional , enfermeira, cirurgiã dentista, farmacêutica).

**Número total de bens domésticos: geladeira, freezer, fogão, TV a cores e branco/preto, rádio, telefone fixo e celular, computador e acesso a Internet.

\section{RESULTADOS}

As variáveis sócio-demográficas estudadas podem ser visualizadas na Tabela 1. Dos 2857 lactentes avaliados, 49,3\% eram do sexo feminino. As mães adolescentes representaram 13,5\% da amostra, enquanto que $7,8 \%$ estudaram até o ensino fundamental e 7,0\% estavam desempregadas no momento da entrevista. O chefe da família foi representado, em $75 \%$ dos casos, pelo pai da criança. De modo geral, a distribuição de renda foi uniforme, com predominância para até seis salários-mínimos. O número de bens domésticos, associado à renda familiar, permitiu classificar a população estudada como de bom nível socioeconômico, de acordo com o Instituto Brasileiro de Geografia e Estatística11 (classe C).

A duração mediana do aleitamento materno pode ser visualizada na Figura 2. A mediana encontrada para o aleitamento materno exclusivo foi de 90 dias (Erro-Padrão - EP 1,2; IC95\% $87,6-92,4)$ e para o aleitamento materno geral foi de 120 dias (EP 1,6; IC95\% 116,9 - 123,1). A prevalência do AME com um mês de vida foi de

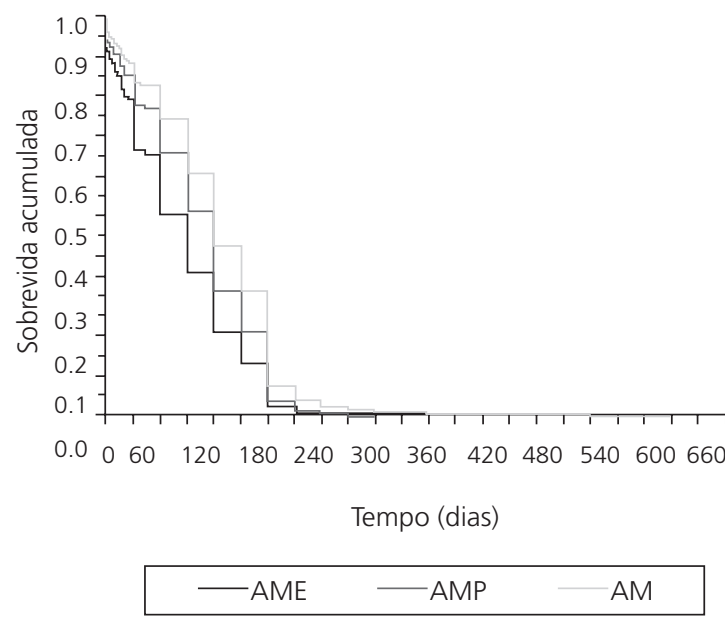

Figura 2. Análise de sobrevida do desmame para aleitamento materno exclusivo, predominante e geral. Campinas (SP), 2004-2005.

Nota: AME: aleitamento materno exclusivo; AMP: aleitamento materno predominante; AM: aleitamento materno geral. 
872 | J.L.D. BERNARDI et al.

$66,2 \%$, decaindo para 2,3\% aos seis meses. Quanto ao aleitamento geral, 83,0\% estavam mamando com um mês de vida, índice que baixou para 7,4\% aos seis meses. Em 1,6\% dos casos as crianças nunca haviam recebido leite de suas mães.

Quando a mediana do aleitamento materno foi correlacionada às variáveis sócio-demográficas (Tabela 2), observou-se que mães adolescentes, assim como as de mais idade, com 5 a 11 anos de estudos, com profissões de nível técnico, ou as desempregadas no momento da entrevista e as que não tinham companheiro, ofereceram leite materno aos seus filhos por menos tempo, tornando-se grupos de risco para o desmame precoce. A tabela permite verificar ainda que os índices medianos do aleitamento não foram diferentes entre os sexos, mas sim entre as crianças de cor da pele diferente: houve significância em relação ao aleitamento materno predominante $(p<0,001)$, pois as negras/pardas são amamentados por menos tempo (60 dias) que as brancas (90 dias).

O resultado do ajuste da análise multivariada de Cox (critério Stepwise), de acordo com a Tabela 3, apontou que a cor da pele da criança, o estado civil e a profissão materna estão associados ao tempo do aleitamento exclusivo. As pardas e negras têm risco $17 \%$ maior de receber aleitamento por menos tempo que as brancas; as muIheres sem companheiro têm risco 17\% maior de amamentar seus filhos por menos tempo; as de profissão de nível técnico têm risco 12\% maior que as do lar e as da área da saúde têm risco $25 \%$ menor que as do lar, de deixar de amamentar exclusivamente. Estes resultados se repetem nos modelos AMP e AM, nos quais se observa que os fatores que garantem a amamentação por mais

Tabela 2. Distribuição do aleitamento materno exclusivo, do aleitamento materno predominante e do aleitamento materno geral, correlacionada às variáveis sócio-demográficas das 2857 crianças. Campinas (SP), 2004-2005.

\begin{tabular}{|c|c|c|c|c|c|c|}
\hline \multirow[b]{2}{*}{ Variáveis } & \multicolumn{6}{|c|}{ M (Mediana em dias) } \\
\hline & AME & $(\mathrm{IC} 95 \%)^{*}$ & AMP & $(I C 95 \%)^{*}$ & AM & $(\text { IC } 95 \%)^{*}$ \\
\hline \multicolumn{7}{|l|}{ Idade materna } \\
\hline Menos de 20 anos & 60 & $(49,8-70,2)$ & 90 & $(79,8-100,2)$ & 120 & $(79,8-100,2)$ \\
\hline De 20 a 34 anos & 90 & $(87,2-92,8)$ & 120 & $(117,4-122,6)$ & 120 & $(117,4-122,6)$ \\
\hline$\geq 35$ & 60 & $(50,9-69,1)$ & 120 & $(109,4-130,6)$ & 120 & $(109,4-130,6)$ \\
\hline \multicolumn{7}{|l|}{ Escolaridade materna } \\
\hline 0 a 4 anos & 90 & $(76,7-103,3)$ & 120 & $(110,8-129,2)$ & 120 & $(110,8-129,2)$ \\
\hline 5 a 8 anos & 60 & $(55,5-64,5)$ & 90 & $(83,8-96,3)$ & 120 & $(83,8-96,3)$ \\
\hline 9 a 11 anos & 60 & $(56,3-63,7)$ & 120 & $(116,7-123,3)$ & 120 & $(116,7-123,3)$ \\
\hline$\geq 12$ & 90 & $(80,0-100,0)$ & 120 & $(113,3-126,7)$ & 120 & $(113,3-126,7)$ \\
\hline \multicolumn{7}{|l|}{ Profissão materna } \\
\hline Do lar & 90 & $(85,8-94,2)$ & 120 & $(116,0-124,0)$ & 120 & $(116,0-124,0)$ \\
\hline Nível superior & 120 & $(94,8-145,2)$ & 120 & $(103,4-136,6)$ & 120 & $(103,4-136,6)$ \\
\hline Área da saúde & 120 & $(98,0-142,0)$ & 120 & $(102,8-137,2)$ & 120 & $(102,8-137,2)$ \\
\hline Nível técnico & 60 & $(56,6-63,4)$ & 90 & $(85,6-94,4)$ & 120 & $(85,6-94,4)$ \\
\hline Sem emprego atualmente & 60 & $(46,8-73,2)$ & 120 & $(109,2-130,8)$ & 120 & $(109,2-130,8)$ \\
\hline \multicolumn{7}{|l|}{ Estado civil mãe } \\
\hline Sem companheiro & 60 & $(56,6-63,4)$ & 90 & $(83,1-96,9)$ & 120 & $(83,1-96,9)$ \\
\hline Com companheiro & 90 & $(87,2-92,8)$ & 120 & $(117,3-122,7)$ & 120 & $(117,3-122,7)$ \\
\hline \multicolumn{7}{|l|}{ Cor de pele da criança } \\
\hline Branca & 90 & $(87,0-93,0)$ & 120 & $(117,3-122,7)$ & 120 & $(117,3-122,7)$ \\
\hline Negra/parda & 60 & $(56,9-63,1)$ & 90 & $(83,7-96,8)$ & 120 & $(83,7-96,8)$ \\
\hline
\end{tabular}

*IC95\%: intervalo de confiança a 95\%.

AME: aleitamento materno exclusivo; AMP: aleitamento materno predominante; AM: aleitamento materno geral. 
Tabela 3. Regressão de Cox Multivariada (critério Stepwise) para o aleitamento materno exclusivo, aleitamento materno predominante, aleitamento materno geral. Campinas (SP), 2004-2005.

\begin{tabular}{|c|c|c|c|c|c|}
\hline Tipo de Aleitamento & Variável & Categorias & $p$-valor ${ }^{*}$ & $\mathrm{HR}^{* *}$ & IC95\%RP $\mathrm{P}^{* * *}$ \\
\hline & Cor da pele & Branca & - & 1,00 & - \\
\hline & & Parda/negra & $<0,001^{*}$ & 1,17 & $1,07-1,28$ \\
\hline & Estado civil & Com companheiro & - & 1,0 & - \\
\hline & & Sem companheiro & $0,004^{*}$ & 1,17 & $1,05-1,30$ \\
\hline \multirow[t]{5}{*}{ AME } & Profissão & Do lar & - & 1,0 & - \\
\hline & Materna & Nível superior & 0,193 & 0,87 & $0,70-1,07$ \\
\hline & & Área da saúde & $0,033^{*}$ & 0,75 & $0,57-0,98$ \\
\hline & & Nível técnico & $0,008^{*}$ & 1,12 & $1,03-1,22$ \\
\hline & & Sem emprego atualmente & 0,626 & 0,96 & $0,82-1,13$ \\
\hline \multirow[t]{9}{*}{ AMP } & Cor da pele & Branca & - & 1,00 & - \\
\hline & & Parda/negra & $0,033^{*}$ & 1,11 & $1,01-1,22$ \\
\hline & Estado civil & Com companheiro & - & 1,00 & - \\
\hline & & Sem companheiro & $0,001^{*}$ & 1,20 & $1,08-1,33$ \\
\hline & Profissão & Do lar & - & 1,00 & - \\
\hline & Materna & Nível superior & 0,736 & 0,96 & $0,78-1,19$ \\
\hline & & Área da saúde & 0,224 & 0,84 & $0,64-1,11$ \\
\hline & & Nível técnico & $0,007^{*}$ & 1,13 & $1,03-1,23$ \\
\hline & & Sem emprego atualmente & 0,968 & 0,99 & $0,85-1,17$ \\
\hline \multirow[t]{9}{*}{ AM } & Cor da pele & Branca & - & 1,00 & - \\
\hline & & Parda/negra & $0,034^{*}$ & 1,11 & $1,01-1,22$ \\
\hline & Estado civil & Com companheiro & - & 1,00 & - \\
\hline & & Sem companheiro & $0,010^{*}$ & 1,16 & $1,04-1,29$ \\
\hline & Profissão & Do lar & - & 1,00 & - \\
\hline & Materna & Nível superior & 0,588 & 1,09 & $0,86-1,31$ \\
\hline & & Área da saúde & 0,426 & 0,89 & $0,67-1,18$ \\
\hline & & Nível técnico & $0,002^{*}$ & 1,16 & $1,06-1,26$ \\
\hline & & Sem emprego atualmente & 0,980 & 1,00 & $0,85-1,19$ \\
\hline
\end{tabular}

${ }^{*} p \geq 0,05$ : estatisticamente significante; ${ }^{* *} \mathrm{HR}$ (Hazard Ratio): razão de risco para o desmame; ${ }^{* * *}$ IC $95 \%$ RP; intervalo de $95 \%$ de confiança para a razão de risco.

AME: aleitamento materno exclusivo; AMP: aleitamento materno predominante; AM: aleitamento materno geral.

tempo são o fato de a mãe viver com seu companheiro, criança de pele branca e as mães com profissão da área da saúde.

\section{DIS CUSS Ã O}

Este é o primeiro trabalho realizado com uma amostra representativa de toda a população de Campinas no que se refere à prevalência do aleitamento na cidade, em crianças menores de dois anos. A mediana encontrada para o aleitamento materno exclusivo foi de 90 dias, porém, em $1,6 \%$ dos casos os lactentes nunca tinham recebido leite de suas mães. As mães, que estu- daram por menos anos, e que não tinham companheiro, amamentaram por menos tempo. Os fatores que interagiram no tempo do aleitamento materno foram a escolaridade, a profissão e o estado civil maternos e a cor da pele da criança. De modo geral, o tempo do AM está menor do que os padrões recomendados internacionalmente; contudo, a mediana do AME em Campinas está maior do que em outros locais do Brasil| ${ }^{5,17-22}$.

Estudos epidemiológicos transversais que trabalham com dados colhidos por meio de entrevistas estão sujeitos a erros ligados a questões como ${ }^{23}$ : memória do entrevistado, informantes secundários, recusas em participar, sigilo das infor- 
$874 \mid$ J.L.D. BERNARDI et al.

mações e tratamento dos dados. Estas dificuldades foram encontradas e, para minimizar a probabilidade destes erros no estudo de Campinas, utilizaram-se técnicas como: a confirmação dos dados (por meio das anotações dos registros contidos no cartão de nascimento, nos dados do SINASC, além da resposta da mãe); entrevistas realizadas com a mãe, de acordo com a data de nascimento das crianças e, deste modo, sendo um evento recente status quo, não chega a afetar a memória do entrevistado, pois foi indagado à mãe o tipo de leite que a criança recebia na época da entrevista; realização de maior número de sorteios em relação ao número amostral e obtenção de perdas menores que $10 \%$, possibilitando alcançar e, até mesmo, ultrapassar o número estatisticamente calculado. Por fim, o tratamento estatístico foi feito com base nas análises uni e multivariadas, objetivando-se minimizar os possíveis dados de confundimento dos resultados.

Diferentemente de outros estudos ${ }^{18-22}$ realizados em áreas mais restritas, neste foi realizado um sorteio aleatório entre os dados que compõem o banco do SINASC, envolvendo as crianças moradoras de todos os bairros da cidade, permitindo a visualização de todo o Município. A mediana do AME para um mês de idade das crianças $(66,2 \%)$ foi maior que a encontrada em pesquisa realizada durante a campanha de vacinação no ano de 200124,25, em unidades básicas de saúde localizadas em quatro regiões de Campinas (AME $46,2 \%)$. No presente estudo, a utilização de um banco de dados como o SINASC propiciou a localização das mães e dos lactentes. O referido banco proporcionou os cálculos estatísticos utilizados na pesquisa e, com 95\% de cobertura, minimizou o viés, pois possibilitou o mapeamento das crianças moradoras de todos os bairros da cidade. As entrevistas foram previamente agendadas por telefone e, na impossibilidade de fazer desta forma, foram marcadas com líderes dos bairros ou nos centros de saúde, o que facilitou o encontro. No momento de responder o questionário, as mães disponibilizaram o cartão de nascimento e as entrevistadoras conferiam os dados com a planilha do
SINASC. Posteriormente, retornavam para a sala de pesquisa da Universidade na qual todo o material preenchido era avaliado pelo supervisor de campo. Todas estas estratégias foram documentadas com a finalidade de minimizar possíveis interferências que pudessem comprometer os resultados da pesquisa. Além disso, outros estudos que estilizaram a entrevista domiciliar como método investigativo sobre dados medianos do aleitamento materno relatam sobre a praticidade e a confiabilidade do questionário ${ }^{26}$.

Dentro do estado de São Paulo, alguns estudos que verificaram a mediana do AME por meio de entrevista domiciliar ou em campanhas de vacinação, encontraram medianas distantes da revelada pelo estudo feito em Campinas: 23 dias em São Paulo ${ }^{26}, 17$ dias em Botucatu ${ }^{27}$ e 18 dias em São José do Rio Preto ${ }^{28}$. Estes dados chamam a atenção para Campinas, que tem índices superiores de $\mathrm{AME}$, em comparação com outras localidades, apesar de estar aquém das recomendações mundiais.

Em relação à etnia, o maior tempo de aleitamento encontrado foi entre as crianças de pele branca, podendo este fato indicar uma tendência de mudança no padrão do comportamento materno em relação à amamentação ${ }^{6}$, pois anteriormente à década de 80 , as crianças de pele negra ou parda eram amamentadas por mais tempo, conforme revela o estudo de Rea ${ }^{29}$. Por outro lado, no estudo de Campinas 715 crianças tinham pele negra/parda, compreendendo $25,1 \%$ da amostra, e o resultado referente ao padrão da amamentação pode ter sido ocorrência do acaso, uma vez que as de pele negra, compreenderam $3,1 \%$ dos lactentes. Por outro lado, a PNDS de $2006^{13}$ mostrou que as mães de cor de pele branca amamentam menos exclusivamente seus filhos que as de pele negra.

Em recente trabalho realizado em uma das regiões menos desenvolvidas socioeconomicamente no interior do Estado de Minas Gerais ${ }^{30}$, 450 crianças menores de 24 meses foram investigadas quanto aos fatores relacionados à duração do aleitamento materno. Os autores associaram 
fatores como o uso de chupetas, a escolaridade paterna e o fato de o pai da criança não residir com a família, ao menor tempo de amamentação. Comparando-se os resultados com o estudo de Campinas, não foram encontradas associações relacionadas à escolaridade paterna e sim à materna e, na ausência do companheiro, as mães amamentaram por menos tempo. Resultados semelhantes também foram encontrados anteriormente por Bueno et al. ${ }^{26}$ e colaboradores na cidade de São Paulo, no ano de 1999. No mesmo período, Alves et al..$^{31}$ e seus colaboradores avaliaram, na cidade de Belo Horizonte - Minas Gerais, os fatores de risco para o desmame entre os anos de 1980 e 2004. O relato das mães sobre a dificuldade inicial para amamentar, foi o maior motivo para a interrupção do aleitamento, nos períodos estudados. Isso mostra que diferentes estratégias deveriam ser abordadas quanto ao apoio e incentivo do aleitamento, levando em consideração a região de moradia e as condições de vida das mães.

Outras regiões do País, como os municípios de Joinville ${ }^{32}$, Cuiabá ${ }^{33}$ e Piauíi ${ }^{34}$, mostram dados sobre a situação do aleitamento materno investigada em campanhas de vacinação. Tais dados identificam que o uso de chupetas, de chás e de mamadeiras, além do trabalho e da escolaridade materna, colabora para que o desmame aconteça, e sugerem ações efetivas municipais e governamentais para o problema.

Em um estudo mais amplo, que analisou os fatores individuais e contextuais que possivelmente se associaram à prática do aleitamento materno exclusivo, mais de 30 mil crianças menores de seis meses foram avaliadas durante a campanha de vacinação realizada no ano de 2001, em 111 municípios do Estado de São Paulo ${ }^{35}$. Os resultados mostraram que a baixa escolaridade materna e o baixo peso ao nascer foram fatores associados ao desmame precoce. Na população de Campinas o tempo de amamentação pode ter sido relacionado com a profissão, pois as mulheres com escolaridade de nível superior, seguidas das que trabalhavam na área da saúde (também no nível superior) foram as que aleitaram mais, embora as mulheres que estudaram menos de quatro anos também tivessem apresentado bons índices de amamentação. As mães que se declararam do lar amamentaram menos, alegando que o bebê chorava muito, o leite era fraco ou o bebê não quis mais (dados encontrados neste estudo, mas não apresentados em tabelas). Estes resultados também foram mostrados em outras pesquisas ${ }^{36-38}$.

Em relação à profissão materna, as hipóteses iniciais deste estudo eram de que os filhos de mães que trabalham em profissões da área da saúde recebessem maior período de aleitamento, pois essas mulheres seriam mais instruídas a respeito de dieta e das técnicas de amamentação. Estes resultados foram confirmados, embora a porcentagem de mães que trabalham na área da saúde não tenha sido a mais prevalente $(2,7 \%$ da amostra).

Em pesquisa realizada na campanha de vacinação do ano de 1999, em uma cidade do interior de São Paulo22, foram estudados 679 lactentes com idade entre 0 e 12 meses. Os resultados revelaram que as mães tinham idade de 25,5 anos; $64,8 \%$ amamentaram exclusivamente seus filhos até um mês e entre quatro e seis meses este número baixou para 9,6\%. No estudo de Campinas, $66,2 \%$ das mães amamentaram exclusivamente seus filhos durante o primeiro mês de vida, índice que baixou pra 3,5\% aos seis meses. O leite em pó foi ingerido em $23,3 \%$ dos casos antes dos três meses de vida, sendo que este fato não foi observado para a introdução do leite fluido. O chá foi introduzido por volta de 120 dias por $18,9 \%$ das mães, porém $13,2 \%$ das crianças receberam chá com poucos dias de vida.

Campinas, mesmo com índices de aleitamento melhores do que em outras regiões do Brasil, ainda não conseguiu alcançar o proposto pela Organização Mundial de Saúde, mesmo possuindo uma estrutura que possibilite atingir a meta. O município tem bons indicadores de saúde, ampla rede de atenção primária, conta com hospitais que possuem Banco de Leite Humano e 
876 | J.L.D. BERNARDI et al.

Hospital Amigo da Criança, tem programas de promoção ao aleitamento materno, distribui cartilhas nas campanhas de vacinação, promove palestras, acompanha as gestantes no pré-natal e as crianças na puericultura. As atividades de orientação às mães têm sido muito importantes, mas não suficientes. $O$ ato de amamentar deve ser amplamente discutido em todas as suas características bio-psicossociais. É preciso aproveitar o potencial de atendimento do sistema de saúde, ouvindo e orientando sobre as questões técnicas, de desconforto ou prazer em relação à amamentação. Algumas propostas de intervenção também poderão ser desenvolvidas junto aos grupos identificados como de risco para o desmame precoce.

Uma vez que há mais de 20 anos o Brasil realiza campanhas de promoção e incentivo ao aleitamento materno e que, ainda assim, o índice nacional está abaixo do preconizado, os resultados do presente estudo mostram que os fatores que garantem a amamentação por mais tempo são o fato de a mãe viver com seu companheiro, criança de pele branca e as mães com profissão da área da saúde. A determinação do perfil do aleitamento no município de Campinas e os fatores associados à sua duração representam a principal contribuição do estudo, uma vez que apontam para a necessidade de repensar estratégias, reavaliar programas, implantar ações mais efetivas para conscientização da população, valorizar a função materna no procedimento e reestruturar as políticas de saúde direcionadas ao aleitamento. Embora melhor que a de outros municípios, a mediana de aleitamento exclusivo está muito aquém da recomendação mundial.

\section{COLABORADORES}

J.L.D. BERNARDI participou integralmente da construção e do trabalho, da coleta de dados e da redação do manuscrito. R.E. JORDÃO realizou levantamento bibliográfico e participou da redação do manuscrito. A.A. BARROS FILHO supervisionou os dados e participou da orientação e da redação do manuscrito.

\section{REFERÊ N CIAS}

1. Brasil. Ministério da Saúde. Manual de promoção do aleitamento materno: normas técnicas. 2a. ed. Brasília; 1997.

2. Almeida JAG, Novak FR. Amamentação: um híbrido natureza-cultura. J Pediatr (Rio J). 2004; 80(5Supl): S119-S25. doi: 10.1590/S0021-75572004000 700002.

3. Ashworth A, Feachem RG. Interventions for the control of diarrhoeal diseases among young children: weaning education. Bull WHO. 1985; 63(6):1115-27.

4. Cushing $A H$, Samet JM, Lambert WE, Skipper BJ, Hunt UC, Young SA, et al. Breastfeeding reduces risk of respiratory illness in infants. Am J Epidemiol. 1998; 147(9):863-70.

5. Kummer SC, Giugliani ERJ, Susin LO, Folletto JL, Lermen NR, Wu VYJ, et al. Evolução do padrão do aleitamento materno. Rev Saúde Publica. 2000; 34(2):143-8. doi: 10.1590/S0034-89102005000 200001.

6. Lamounier JA. Tendências do aleitamento materno no Brasil. Rev Méd Minas Gerais. 1999; 9(2): 59-65.

7. World Health Organization. United Nations Children's Foundations. Innocent Declaration on the Protection, Promotion and Support of Breastfeeding in the 1990's: a Global Initiative. Florence, (Italy): WHO; 1990 [cited 2007 Aug 9]. Available from: <http://www.unicef.org>.

8. Giugliani ERJ. Rede nacional de Banco de Leite Humano no Brasil: tecnologia para exportar. J Pediatr. 2002; 78(3):183-4. doi: 10.1590/S0021-75 572002000300001.

9. World Health Organization. Evidence for the ten steps to successful breastfeeding division of child health and development. Geneva: WHO; 1998.

10. World Health Organization. The Optimal duration of exclusive breastfeeding [note for the press, 2 April of 2001]. Geneva: WHO; 2001.

11. Instituto Brasileiro de Geografia e Estatística. Pesquisa nacional por amostra de domicílios: acesso e utilização de serviços de saúde (documento 44). Rio de Janeiro; 1998 [acesso 2006 ago 9]. Disponível em: <http://www.ibge.gov.br>.

12. Sistema estadual de análise de dados de São Paulo: 1998/2002. [acesso 2006 ago 9]. Disponível em: $<$ http://www.seade.gov.br>.

13. Brasil. Ministério da Saúde. Pesquisa nacional de demografia e saúde da criança e da mulher. Relatório final. Brasília: Ministério da Saúde; 2006 [acesso 2008 out 29]. Disponível em: <http:// 
bvsms.saude.gov.br/bvs/pnds/img/relatorio_ final_pnds2006.pdf>.

14. Silva AAM, Ribeiro US, Borba JRAF, Coimbra LC, Silva RA. Avaliação da qualidade dos dados do sistema de informação sobre nascidos vivos em 1997-1998. Rev Saúde Pública. 2001; 35(6): 508-14. doi: 10.1590/S0034-89102001000600 003.

15. Dean AG, Dean JA, Coulombier D, Brendel KA, Smith DC, Burton A, et al. Epi Info [computer program]. Version 6,0: a word processing, database, and statistic program for epidemiology on micro-computers. Atlanta: Centers of Disease Control and Prevention; 1994.

16. Statistical Package for Social Science for windows [computer program]. Release 7.0. Chicago (IL): 1996.

17. Brasil. Ministério da Saúde. Prevalência do aleitamento materno nas capitais brasileiras e no Distrito Federal. Brasília: Ministério da Saúde; 2001.

18. Ricco RG, Ciampo LAD, Almeida CAN, Daneluzzi JC. Aleitamento exclusivamente ao seio, morbidade e utilização de serviço pediátrico em unidade básica de saúde. Pediatria (São Paulo). 2001; 23(2): 193-8.

19. Oliveira MIC, Camacho LAB. Impacto das unidades básicas de saúde na duração do aleitamento materno exclusivo. Rev Bras Epidemiol. 2002; 5(1): 41-51. doi: 10.1590/S1415-790X20020001000 06.

20. Mascarenhas MLW, Albernaz EP, Silva MB, Silveira RB. Prevalência do aleitamento materno nos três primeiros meses de vida e seus determinantes no sul do Brasil. J Pediatr (Rio de Janeiro). 2006; 82(4): 289-94. doi: 10.1590/S0021-75572006000500 011.

21. Ferreira L, Parada CMGL, Carvalhaes MABL. Tendência do aleitamento materno em município da região centro-sul do estado de São Paulo: 19951999-2004. Rev Nutr. 2007; 3(20):265-73. doi: 10.1590/S1415-52732007000300005.

22. Audi CAF, Correa MAS, Latorre MRDO. Alimentos complementares e fatores associados ao aleitamento materno e ao aleitamento materno exclusivo em lactentes até 12 meses de vida em Itapira, São Paulo. Rev Bras Saúde Mater Infant. 2003; 3(1): 85-93. doi: 10.1590/S1519-38292003000100 011.

23. Viacava F. Informações em saúde: a importância dos inquéritos populacionais. Ciência Saúde Coletiva. 2002; 7(4):607-21. doi: 10.1590/S1413-81 232002000400002
24. Claro RM, Buarraj MC, Silva AT, Zoldan CM, Moura EC. Prevalência e duração da amamentação em crianças de 0 a 2 anos na periferia de Campinas, São Paulo, Brasil. Rev Ciên Méd. 2004; 13(4): 337-46.

25. Cecchetti DFA, Moura EC. Prevalência do aleitamento materno na região noroeste de Campinas, São Paulo, Brasil, 2001. Rev Nutr. 2005; 18(2): 201-8. doi: 10.1590/S1415-527320050002 00004.

26. Bueno MB, Souza JMP, Souza SB, Paz SMRS, Gimeno SGA, Siqueira AAF. Riscos associados ao processo de desmame entre crianças nascidas em hospital universitário de São Paulo, entre 1998 e 1999: estudo de coorte prospectivo do primeiro ano de vida. Cad Saúde Pública. 2003; 19(5): 1453-60. doi: 10.1590/S0102-311X20030005 00024.

27. Carvalhaes MABL, Parada CMGL, Manoel CM, Venâncio SY. Diagnóstico da situação do aleitamento materno em área urbana do Sudeste do Brasil: utilização de metodologia simplificada. Rev Saúde Pública. 1998; 32(5):430-6. doi: 10.1590/ S0034-89101998000500005.

28. Figueiredo MG, Sartorelli DS, Zan TAB, Garcia E, Silva LC, Carvalho FLP, et al. Inquérito de avaliação rápida das práticas de alimentação infantil em São José do Rio Preto, São Paulo, Brasil. Cad Saúde Pública. 2004; 20(1):172-9. doi: 10.1590/S0102-31 $1 \times 2004000100033$.

29. Rea MF. Avaliação das práticas diferenciais de amamentação: a questão da etnia. Rev Saúde Pública. 1994; 5(28):365-372. doi: 10.1590/S0034-8 9101994000500010 .

30. Silveira FJF, Lamounier JA. Fatores associados à duração do aleitamento materno em três municípios na região do alto Jequitinhonha, Minas Gerais, Brasil. Cad Saúde Pública. 2006; 22(1): 69-77. doi: 10.1590/S0102-311X20060001000 08.

31. Alves CRL, Goulart EMA, Colossimo EA, Goulart LMHF. Fatores de risco para o desmame entre usuárias de uma unidade básica de saúde de Belo Horizonte, Minas Gerais, Brasil, entre 1980 e 2004. Cad Saúde Pública. 2008; 24(6):1355-67. doi: 10.1590/S0102-311X2008000600016.

32. Franco SC, Nascimento MBR, Reis MAM, Issler H, Grisi SJFE. Aleitamento materno exclusivo em lactentes atendidos na rede pública do município de Joinville, Santa Catarina, Brasil. Rev Bras Saúde Mater Infant. 2008; 8(3):291-97. doi: 10.1590/ S1519-38292008000300008. 
33. França GVA, Brunken GS, Silva SM, Escuder MM, Venâncio SI. Determinantes da amamentação no primeiro ano de vida em Cuiabá, Mato Grosso. Rev Saúde Pública. 2007; 41(5):711-8. doi: 10.15 90/S0034-89102007000500004.

34. Ramos CV, Almeida JAG, Alberto NSMC, Teles JBM, Saldiva SRDM. Diagnóstico da situação do aleitamento materno no Estado do Piauí, Brasil, Cad Saúde Pública. 2008; 24(8):1753-62. doi: 10.1590/ S0102-311X2008000800004.

35. Venâncio SI, Monteiro CA. Individual and contextual determinants of exclusive breast-feeding in São Paulo, Brazil: a multilevel analysis. Public Health Nutr. 2006; 9(1):40-6.

36. Volpini CCA, Moura EC. Determinantes do desmame precoce no Distrito Noroeste de Campinas.
Rev Nutr. 2005; 18(3):311-9. doi: 10.1590/S14 15-52732005000300003.

37. Silva SM, Brunken GS, França GVA, Escuder MM, Venâncio SI. Evolução do aleitamento materno em uma capital da região Centro-Oeste de Brasil entre 1999 e 2004. Cad Saúde Pública. 2007; 23(7): 1539-46. doi: 10.1590/S0102-311X20070007 00004.

38. Nejar FF, Segall-Corrêa AM, Rea MF, Vianna RPT, Panigassi G. Padrões de aleitamento materno e adequação energética. Cad Saúde Pública. 2004; 20(1):64-71. doi: 10.1590/S0102-311X20040001 00020 .

Recebido em: 10/12/2007

Versão final reapresentada em: 5/11/2008 Aprovado em: 25/3/2009 